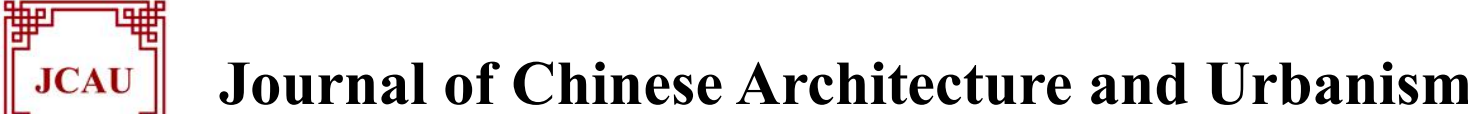

\section{Influential Theories and Works for Contemporary Chinese Urban Planning and Design (1920-2020)}

\author{
Karine Dupre $^{1}$, Hing-Wah Chau ${ }^{2}$, Bixia $\mathrm{Xu}^{3}$ \\ ${ }^{1}$ School of Engineering and Built Environment, Griffith University, Gold Coast, QLD, 4215, \\ Australia \\ ${ }^{2}$ College of Engineering and Science (Built Environment Group), Victoria University, Victoria, \\ Australia \\ ${ }^{3}$ Asia Pacific Agri-Corp (Projects) Pty Ltd, Australia
}

Corresponding author: Karine Dupre, School of Engineering and Built Environment, Griffith University, Gold Coast, QLD, 4215, Australia. Email: k.dupre@griffith.edu.au

Citation: Dupre K, Chau H-W, Xu B, 2021, Influential Theories and Works for Contemporary Chinese Urban Planning and Design (1920-2020). Journal of Chinese Architecture and Urbanism, 3(1): 1080. http://dx.doi.org/10.36922/jcau.v3i1.1080

Keywords: urban planning and design, China, heritage, rural urbanization, large-scale urban planning

This editorial belongs to the Special Issue: Influential theories and works for contemporary Chinese urban planning and design (1920-2020)

Cities and their footprints not only reveal ways of planning, designing, and building from the past, but also inform on today's experience and projects for the future. As such, their physicality is embedded in ideas, as well as social and cultural meanings that fluctuate with time. This special issue addresses this complexity in exploring the tangible and intangible features of influential theories and works for contemporary Chinese urban planning and design (1920-2020). Although the starting date could be debated (see, for example, the insightful article of Liu
[1]), 2020 was actually our starting point as there is little argument that there is a before and after the pandemic for our cities. The already numerous published scholarships questioning urban planning and design, both at practices and users' level, show that we can forecast drastic changes in planning and designing our cities, placemaking, and sense of identity and belonging.

On the other hand, with a continuous fast-paced urbanization, China remains a fascinating venue of experimentations and research for architects, planners, urban

Copyright: () 2021 Dupre, et al. This is an open-access article distributed under the terms of the Attribution-NonCommercial 4.0 International 4.0 (CC BY-NC 4.0), which permits all non-commercial use, distribution, and reproduction in any medium provided the original work is properly cited. 
designers, and landscape architects alike. It was timely therefore to examine the historical and theoretical influences, both nationally and internationally that have contributed to shaping contemporary Chinese urban planning and design. Three main themes emerged that organise the content of this special issue: planning and heritage, rural urbanization, and the effects of large-scale urban planning. These topics unsurprisingly confirm the weight of external influences and political regimes, as described by Shi ${ }^{\text {[2] }}$ for instance.

In the first article under the planning and heritage topic, Shiran Geng, Hing-Wah Chau, Tian Wang and Se Yan argue that Harbin (Heilongjiang Province) was one of the most crucial junction cities as it was located on the Chinese Eastern Railway connecting Russia and China. Through a review of existing literature and archive collections, map analysis and field observations, four selected districts of Harbin under Russian influence were scrutinised insightfully from multiple perspectives, including European planning strategies and garden city movement. Based on the discussion, this article provides heritage conservation approaches and recommendations which can be transferable to other colonial cities.

In the second article, Yang Liu, Karine Dupre and Xin Jin use Dalian (Liaoning Province) as a case study to reveal the international and national influences that have shaped the city. The methodology is based on archival investigation and urban morphology analysis, showing that the Dalian of today owes much of its contemporary fabric to a unique combination of urban planning theories. The latter not only demonstrates the impact of colonial history, but also the circulation of ideas and knowledge-sharing in contemporary China. Most importantly, this article evidences how urban planning has been instrumentalized by different regimes, and how the global competition, often symbolized by tourism development, raises issues of equity, inclusiveness, and identity.

The next two papers are focused on rural urbanization. In her article, Ava Lynam focuses on rural-urban transformations and specifically Characteristic Towns which aim to attract investment to rural areas and optimizing the planning of urban agglomerations. Investigating the case study of Smart Moulding Town (Zhejiang Province) through a mixed method, the author highlights that current framework needs to be challenged at the institutional level to address the obsolete rural-urban dichotomy, while rural communities are strained under the weight of changes and adaptations they must undertake. Additionally, this contribution shows that the rural urbanization process often leaves important environmental and social issues unaddressed, thus emphasizing the need for a shift in planning regarding areas deemed of interest.

With the case study of Simatai Village (Beijing), Linlin Dai, Jiaming Fu, Xiao Rong, Dong Li and Ling Yang discuss the radical spatial and social transformations that occur when tourism is introduced in planning to increase economic growth and urbanization. Based on a morphological analysis, the study shows that in many aspects Simatai is a success story as economical, social, and environmental benefits can be evidenced. However, the authors also underline how there are some fundamental questions being raised regarding authenticity, as well as community engagement and empowerment, thus questioning the long-lasting top-down governmental approach.

The last two articles are focused on the effects of large-scale urban planning. The contribution by Han Cheng and Julian Worrall argue that urban public art is an important means to understand cultural policies - or their lack of -, as well as the drivers for planning and economic growth. Based on two case studies, Beijing and Shanghai, the authors reconstruct the history of contemporary public art in China and demonstrate how there is a clear relationship with urbanization and planning strategies, yet often subdued by a political agenda that leaves little room to cultural creativity and empowerment. 
The last article by Raffaele Pernice is based on a case study in Suzhou (Jiangsu Province), investigating new high-rise residential urban superblocks. Through a mixed method including interviews and spatial analysis, the author sheds light on the historical influences that generated this typical urban planning and modernization, but also unfolds the pros and cons of such large-scale projects. Some elements emerged in the findings that call for future changes, to maintain the quality of life and the sustainability of the natural environment. Additionally, the question is raised about creativity and innovation to better reflect the diversity in communities.

In conclusion, we hope this special issue contributes to new knowledge and beyond the limitations of single case studies, opening up new windows as more scholarship is definitely needed to draw an accurate picture of the influential theories and works for contemporary Chinese urban planning and design. This is necessary at a time of the 2030
Agenda for Sustainable Development because a good understanding of all these is key for a prosperous future.

\section{CONFLICT OF INTEREST}

The authors are the guest editors of the Special Issue: Influential theories and works for contemporary Chinese urban planning and design (1920-2020). K.D. and H-W.C. are co-authors of two articles published in this Special Issue, and their papers were handled by the non-author guest editors and the Editor-in-Chief.

\section{REFERENCES AND NOTES}

[1] Liu Y. A chronology of the field of modern Chinese architectural history, 19862012 [J]. Frontiers of Architectural Research, 2013, 2(2): 191-208.

[2] Shi M. History of urban planning (Chinese), International Encyclopedia of the Social \& Behavioral Sciences. 2001 Elsevier Science Ltd., 16044-16047. 